Société d'histoire de la révolution de 1848 et des

révolutions du XIXe siècle

49 | 2014

1814-1815. Expériences de la discontinuité

\title{
Fraternités libérales et insurrections nationales : Naples et l'Espagne, 1820-1821
}

Liberal brotherhood and national insurrections: Naples and Spain, 1820-1821

Liberale Bruderschaften und nationale Erhebungen: Neapel und Spanien, 1820-1821

\section{Pierre-Marie Delpu}

\section{(2) OpenEdition Journals}

Édition électronique

URL : http://journals.openedition.org/rh19/4762

DOI : $10.4000 /$ rh 19.4762

ISSN : $1777-5329$

Éditeur

La Société de 1848

Édition imprimée

Date de publication : 1 décembre 2014

Pagination : 195-213

ISSN : 1265-1354

Référence électronique

Pierre-Marie Delpu, « Fraternités libérales et insurrections nationales : Naples et l'Espagne, 1820-1821 », Revue d'histoire du XIXe siècle [En ligne], 49 | 2014, mis en ligne le 01 décembre 2014, consulté le 01 mai 2019. URL : http://journals.openedition.org/rh19/4762 ; DOI : 10.4000/rh19.4762 


\section{PIERRE-MARIE DELPU}

\section{Fraternités libérales et insurrections nationales: Naples et l'Espagne, 1820-1821}

Le 2 juillet 1820, quelques militaires politisés se soulèvent dans la garnison de Nola, en périphérie de Naples, pour réclamer l'adoption de la constitution des Cortès de Cadix de 1812 par le roi Ferdinand I Ier des Deux-Siciles. Trois mois après, l'un de leurs chefs, le général Guglielmo Pepe, l'un des principaux vétérans napolitains de la Grande Armée, déclare au jeune Parlement napolitain vouloir faire du royaume "une seconde île de León " ${ }^{1}$, suggérant que la révolution, encore à l'œuvre, vise à convertir le royaume méridional à la modernité politique incarnée par la révolution d'Espagne. En faisant référence au lieu symbolique où ont débuté les deux soulèvements gaditains de 1812 et de 1820, il revendique les parentés entre les insurrections d'Espagne et de Naples, faisant de la première un "archétype révolutionnaire» qui aurait dicté le déroulement de la seconde ${ }^{2}$. Si les libéraux napolitains empruntent en effet aux révolutionnaires espagnols leur constitution, ils puisent également dans l'expérience espagnole un répertoire d'action, des images et des langages politiques. Parmi ceux-ci émerge alors dans le lexique politique napolitain la catégorie "libéral», désignant, sans théorie ni nuance, toutes les formes de l'opposition au régime bourbonien considéré comme despotique et de l'engagement patriotique éclairé.

Ce processus d'importation apparent a renforcé, du point de vue des observateurs étrangers marqués par la peur de la contagion révolutionnaire, l'idée que la révolution napolitaine était illégitime parce qu'imposée par des militaires en rébellion et fondée sur des réseaux clandestins capables de déclencher une insurrection généralisée en Europe. Plénipotentiaire français au congrès de Vérone (1823) chargé de définir la politique de la SainteAlliance à l'égard du trienio libéral espagnol, Chateaubriand en souligne le rôle matriciel au regard des révolutions italiennes de 1820-1821 : «Les propagandistes de l'intérieur de l'Espagne s'étaient réjouis en voyant leur œuvre

1. Guglielmo Pepe, Rapport au Parlement, Naples, 2 octobre 1820 ; cité par Ruggero Moscati, Guglielmo Pepe, Roma, Vittoriano, 1938, volume 1, pièce 135, p. 112-114.

2. L'expression est utilisée par Irene Castells Oliván, «Le libéralisme insurrectionnel espagnol, 1814-1830", Annales historiques de la Révolution française, n 336, 2004/2, p. 221-233. 
s'étendre au-dehors; la Constitution de Cadix avait été imposée à Naples : Naples en fut pour son caprice, il lui fallut retourner à son soleil et à ses fleurs " ${ }^{3}$. Par cette observation critique, il dénonce la légèreté et l'irréflexion avec lesquelles le modèle espagnol aurait influencé les révolutionnaires napolitains, mais n'aurait pas pour autant permis l'implantation durable d'un régime libéral à Naples. Le constat est classique chez les observateurs étrangers : peu après l'éclatement de la révolution napolitaine en 1820 , le diplomate français Remsberg la réduit à une "grotesque imitation de celle d'Espagne $»^{4}$.

L'exportation de la révolution espagnole en Italie est connue de l'historiographie, traditionnellement attentive à la relation hispano-italienne, même si elle ne s'est pas penchée spécifiquement sur le cas napolitain 5 . Après avoir tenté d'expliquer la place du modèle espagnol dans les débuts du Risorgimento italien $^{6}$, les historiens insistent, plus généralement, sur les aspects transnationaux des révolutions du XIX ${ }^{\mathrm{e}}$ siècle et accordent une place importante à l'Italie préunitaire, en étudiant à nouveaux frais le rôle politique des exilés italiens ou l'existence d'une culture constitutionnelle partagée 7 . La méthode des circulations et des transferts révolutionnaires a montré à quel point l'Europe du XIX siècle était un "espace de communication" transnational fournissant un cadre favorable à des projets internationalistes modernisateurs, particulièrement au moment des révolutions libérales et démocratiques de 1830 et de $1848^{8}$. La Pepa de Cadix est l'objet de réévaluations importantes qui en ont

3. François-René de Chateaubriand, Le Congrès de Vérone, Paris, Delloye, 1838, p. 21.

4. Archives diplomatiques, Paris, Mémoires et documents, Naples, 11, Remsberg, Deuxième mémoire sur l'Italie, juillet 1820. 1950.

5. Giorgio Spini, Mito e realtà della Spagna nelle rivoluzioni italiane del 1820-21, Roma, Perrella,

6. L’ouvrage ancien de Juan Ferrando Badía, La Constitución española de 1812 en los comienzos del "Risorgimento", Madrid-Roma, Consejo Superior de Investigaciones, 1959, relève de l'historiographie juridique et constitutionnelle. Les traitements du sujet par les historiens du politique, à la fois espagnols et italiens, sont postérieurs. Parmi une riche littérature et côté italien : Antonino De Francesco, «La costituzione di Cadice nella cultura politica italiana del primo Ottocento ", in Antonino De Francesco [dir.], Rivoluzione e costituzioni. Saggi sul democratismo politico nell'Italia napoleonica. 1796-1821, Naples, ESI, 1996; Paolo Colombo, "Costituzione come ideologia. Le rivoluzioni italiane del 182021 e la costituzione di Cadice", in José Maria Portillo Valdés [dir.], La Nazione cattolica. Cadice 1812 : una costituzione per la Spagna, Manduria, Lacaita, 1998, p. 129-157; Maria Sofia Corciulo, "La circolazione del modello spagnolo in Italia (1820-1821)», in Fernanda Mazzanti Pepe [dir.], Culture costituzioni a confronto, Gênes, Name, 2005, p. 129-148.

7. Sur les exilés, $c f$. Agostino Bistarelli, Los exiliados italianos en el trienio liberal, thèse sous la direction d'Antonio Moliner Prada, Universidad Autònoma de Barcelona, 2009, et Maurizio Isabella, Risorgimento in Exile. Italian Emigrés and the Liberal International in the Post-Napoleonic Era, Oxford, Oxford University Press, 2009. Sur les constitutions, cf. Jens Späth, Revolution in Europa 1820-1823. Verfassung und Verfassungskultur in den Königreichen Spanien, beider Sizilien und Sardinien-Piemont, Cologne, SH-Verlag, 2012. Le transfert de la révolution espagnole en Piémont a fait l'objet d'un travail spécifique : Gonzalo Butrón Prida, Nuestra sagrada causa. El modelo gaditano en la revolución piamontesa de 1821, Cadix, Fundación municipal de cultura, 2006.

8. L'expression est empruntée à Dieter Langewiesche, "Kommunikationsraum Europa : Revolution und Gegenrevolution", in Dieter Langewiesche [dir.], Demokratiebewegung und Revolution. 1847 bis 1849. Internationale Aspekte und europäische Verbindungen, Karlsruhe, Braun, 1998, p. 11-35. Cf. également Sylvie Aprile, Jean-Claude Caron, Emmanuel Fureix [dir.], La liberté guidant les peuples. Les révolutions de 1830 en Europe, Seyssel, Champ Vallon, 2013. 
montré la signification juridique et politique pour refonder une monarchie en crise et en ont saisi la réception coloniale et globale, sans qu'en soient interrogés dans ce sens les prolongements dans les révolutions de Naples et de Piémont ${ }^{9}$. Le libéralisme napolitain, qui émerge alors comme courant politique hétéroclite regroupant les opposants aux Bourbons, les héritiers des Lumières et des révolutions, qu'ils soient républicains ou partisans de la monarchie constitutionnelle, s'insère pourtant dans un cadre plus large, qualifié d' "internationale libérale» et dont les dimensions correspondent, globalement, à celles de l'espace euro-américain des révolutions "occidentales» de la fin du XVIII et du début du XIX ${ }^{e}$ siècle. L'existence de caractères politiques, sociaux et culturels communs permet d'apparenter, à la suite de travaux récents, le royaume méridional aux "périphéries atlantiques» qui ont subi les répercussions des révolutions globales des années 1770-182010, confirmant les parentés entre les révolutions occidentales au sens large et le libéralisme napolitain émergent.

Les premiers mois de l'année 1820, au cours desquels éclate la révolution espagnole, voient se développer à Naples d'importantes sympathies hispanophiles, de manière soudaine, à l'appui de circulations transnationales d'hommes et d'idées. Les premiers jours de la révolution ont vu se déployer, principalement à destination des provinces faiblement tenues par l'armée, des circuits de politisation spécifiques qui ont permis le développement d'un relatif consensus autour du texte espagnol. Mais le texte n'a pas moins fait l'objet de débats importants, autant chez les juristes qu'au sein même de l'opinion publique napolitaine, discutant de son adéquation aux conditions politiques et sociales du royaume des Deux-Siciles.

\section{L'Espagne À Naples, du Malgoverno au modèle politique}

L'engouement pour la constitution de Cadix qu'ont exprimé les Napolitains en insurrection à l'été 1820 paraît surprenant dans un royaume occupé pendant plusieurs siècles par la monarchie espagnole et dont l'argument antiespagnol a constitué l'un des principaux facteurs identitaires ${ }^{11}$. Au début du $\mathrm{XIX}^{\mathrm{e}}$ siècle encore, des libéraux comme Vincenzo Cuoco, Pietro Colletta ou Luigi Blanch continuent d'associer l'Espagne au malgoverno qu'elle a imposé

9. Par exemple José Maria Portillo Valdés, «La constitución en el Atlántico hispano, 18081824», Fundamentos, n 6, 2010, p. 123-178; Carlos María Rodriguez López Brea, «La Constitución de Cádiz y el proceso revolucionario en las dos Sicilias (1820-1821) ", Historia contemporánea, ${ }^{\circ}$ 47, 2013, p. 561-594.

10. Cf. notamment Rocco W. Ronza, «Appunti per un modello di modernizzazione politica della "periferia occidentale" : dalle Rivoluzioni atlantiche alla genesi dello Stato liberale (17601859) », Annali di storia moderna e contemporanea, 2013, n 1, p. 73-98.

11. Aurelio Musi [dir.], Alle origini di una nazione. Antispagnolismo e identità italiana, Milan, Guerini, 2004. 
pendant plusieurs siècles à ses possessions d'Italie méridionale. Un texte comme le Saggio storico sulla rivoluzione napoletana del 1799, publié en 1801, fait de l'ancien occupant le parangon de la réaction politique par son système absolutiste et son histoire moderne marquée par le poids de l'Inquisition. La même littérature impute à la monarchie espagnole la répression de la révolte de Masaniello à Naples en 1648, érigée en lieu de mémoire du patriotisme napolitain ${ }^{12}$. C'est précisément au nom de ces arguments historiques et politiques que s'est développée l'hostilité envers la communauté espagnole de Naples au début du XIX ${ }^{\mathrm{e}}$ siècle $^{13}$. Dès lors, l'actualité politique espagnole a été l'objet d'une attention limitée : la constitution de Cadix n'a été que très peu commentée à Naples lors de son adoption en 1812, malgré la crise dans laquelle s'engageait le royaume de Murat, affaibli à la fois par la campagne de Russie et par les oppositions montantes à gauche qui réclamaient une constitution libérale. La référence espagnole est alors presque absente du paysage culturel napolitain : à l'exception de quelques traductions du Don Quichotte de Cervantès, aucun ouvrage espagnol ou évoquant l'Espagne n'est édité dans le royaume.

L'explosion des publications consacrées à la vie politique espagnole est pourtant significative à partir du début de la révolution de Riego dans les premiers mois de 1820. Les événements d'Espagne sont très suivis à Naples, largement relayés par la presse. Dans l'organe officiel du gouvernement révolutionnaire, le Giornale costituzionale del Regno delle Due Sicilie, la répartition des notices d'information montre le primat de l'actualité espagnole, à la fois métropolitaine et coloniale. Sur les deux premiers mois de la publication du journal (juillet et août 1820), 34\% d'entre elles concernent l'Espagne (colonies comprises), contre $23 \%$ pour l'actualité britannique, $18 \%$ pour la France ou encore $5 \%$ pour les autres États italiens. À partir du moment où le roi jure fidélité à la constitution le 13 juillet, le même journal publie en feuilleton, tous les jours, le texte de la constitution publié dans la traduction du chanoine Juan Francisc Masdeu, historiographe renommé qui a déjà traduit des textes poétiques et des sommes historiographiques de l'espagnol vers l'italien, et qui fait alors référence ${ }^{14}$. Il s'agit de la seule version du texte gaditain connue en Italie, tirée à huit éditions en 1814 dont une à Messine et deux à Rome : aucune n'a été imprimée dans le royaume de Naples. Le texte aurait cependant déjà été l'objet de revendications de la part des libéraux

12. Alain Hugon évoque, à propos des récupérations successives de cet épisode de l'histoire politique méridionale, un "palimpseste révolutionnaire» déterminant dans l'évolution du royaume jusqu'à son intégration à l'Italie unitaire. Cf. Naples insurgée, 1648. De l'événement à la mémoire, Rennes, Presses Universitaires de Rennes, 2011.

13. Sur cet aspect, $c f$. Raffaella Salvemini, «Gli Spagnoli a Napoli al tempo dei Napoleonidi (1806-1815). Le ragioni di una débâcle economica e politica", Mélanges de l'École française de Rome. Italie et Méditerranée, 1999, volume 111/2, p. 683-719.

14. Costituzione Politica della Monarchia Spagnuola promulgata in Cadice nel marzo del 1812. Tradotta in italiano da G.F. Masdeu nel gennajo del 1814, Roma, Salvioni, 1814, plusieurs éditions. 
napolitains, ce qui laisse supposer une circulation antérieure aux révolutions de $1820^{15}$.

La circulation des hommes, d'autre part, a contribué de façon déterminante à la diffusion du modèle insurrectionnel espagnol. Les observateurs internationaux s'inquiètent, très tôt, de circulations clandestines susceptibles de propager la révolution : le consul britannique en poste dans le royaume, William A'Court, s'alarme dès le 31 mars 1820 des effets produits à Naples par la révolution d'Espagne, notant une agitation non négligeable et la revendication d'une constitution libérale par les carbonari. En juin, il relève plusieurs arrestations de carbonari napolitains à cause de la fermentation provoquée par les nouvelles d'Espagne. Ce sont les mêmes événements qui produisent, le 22 juin, de violentes manifestations à Salerne conduisant la monarchie bourbonienne à changer deux des intendants en poste dans la province que commande la ville, le Principat Citérieur ${ }^{16}$. Le républicain molisan De Attellis mentionne, tout en restant relativement vague, des rapports d'interconnaissance entre plusieurs prêtres du Principat Citérieur, autour de Salerne, affiliés à la Charbonnerie, et des révolutionnaires de la région de Valence qui auraient permis la diffusion du texte à l'échelle locale, un an avant le déclenchement de la révolution. Un prêtre libéral très influent dans les réseaux carbonari locaux, Matteo Farro, curé de Bellosguardo, a en effet diffusé la nouvelle d'une révolte libérale survenue à Valence, de retour d'un voyage en Espagne en $1819^{17}$. Les mêmes solidarités se retrouvent pendant la révolution où la correspondance de Guglielmo Pepe avec plusieurs sociétés patriotiques espagnoles est régulièrement reproduite par la presse napolitaine ${ }^{18}$.

La promotion du modèle insurrectionnel gaditain n'est cependant pas limitée aux réseaux clandestins de la Charbonnerie. On trouve sur le territoire méridional peu d'Espagnols politisés en lien avec les carbonari napolitains, mais les milieux diplomatiques occupent une fonction non négligeable de passeurs. C'est le cas de l'ambassadeur espagnol Luis de Onís, en poste à Naples depuis 1819, qui entretient des liens épistolaires réguliers avec le général Guglielmo Pepe ${ }^{19}$. Cette correspondance se développe surtout au

15. Le républicain molisan Orazio De Attellis signale, dans une reconstitution rédigée en exil à Barcelone en 1821, que des carbonari auraient envoyé en 1817 au roi Ferdinand I $\mathrm{I}^{\text {er }}$ et à ses ministres plusieurs copies du texte espagnol qu'ils souhaitaient voir adopté à Naples, information que les archives bourboniennes ne permettent pas de confirmer, en dépit du suivi très régulier de l'esprit public dans la totalité du royaume. Cf. Orazio De Attellis, L'Ottimestre costituzionale delle Due Sicilie, Biblioteca Nazionale di Napoli (notée par la suite BNN), mss. V, A, 47-2.

16. The National Archives, Kew (notées par la suite TNA), FO 605/185, 2. 1820, Foreign. Correspondance de William A'Court avec le Foreign Office (19, 31 mars 1820; 32, 13 juin 1820; 34, 22 juin 1820).

17. Biblioteca Nazionale Centrale, Roma, Misc. Ris., f. 106.

18. Voir notamment BNN, SM, Fogli volanti, SQ IV, L. 28 n. 175, lettera de'Carbonari di Spagna al Generale Pepe. Società patriottica degli amanti dell'ordine costituzionale, Madrid, 3 août 1820 .

19. Voir Archivio Histórico Nacional, Madrid (noté par la suite AHN), leg. 5675. La correspondance est pour partie retranscrite dans les annexes de l'ouvrage de Giorgio Spini, Mito e 
moment de l'expédition punitive engagée par l'Autriche dans les premiers mois de 1821, où Pepe conduit l'armée constitutionnelle dans les Abruzzes pour défendre le régime en place. De façon très régulière, il informe l'ambassadeur espagnol de l'évolution de la situation politique et militaire des Napolitains face à l'Autriche et souligne la forte sensibilité patriotique du peuple napolitain. À cet égard, Onís constitue un interlocuteur direct pour les chefs libéraux napolitains. Ces liens lui valent d'être considéré, dès les premières émeutes de juillet 1820, comme l'un des fauteurs de la révolution de Naples : les autorités bourboniennes et une partie de la population du royaume supposent que la révolution a été imposée depuis Madrid par l'intermédiaire d'Onís au nom de parentés historiques et culturelles entre les deux États ${ }^{20}$. Si l'accusation, fondée sur la rumeur, est évidemment exagérée, il n'en est pas moins vrai qu'Onís a indéniablement joué le rôle d'un passeur entre les deux révolutions. Les libéraux napolitains ont donc connaissance de la révolution d'Espagne et de son scénario, mais le texte gaditain semble très peu connu pour lui-même, en dépit de sa très forte circulation. Il est plutôt le support de représentations qui cherchent à légitimer les événements de Naples par la référence au précédent espagnol.

\section{IMITER LA RÉVOLUTION D'ESPAGNE : OPPORTUNISME POLITIQUE ET FRATERNITÉS RÉVOLUTIONNAIRES}

Le choix du texte de Cadix par les révolutionnaires napolitains est l'objet d'une importante littérature de justification qui passe à la fois par la presse et par les débats parlementaires. La constitution semble avoir été très peu lue et, à l'exception de quelques auteurs prônant le bicaméralisme, ses aspects proprement institutionnels n'ont fait l'objet que de très rares commentaires édités alors même que des collections de constitutions ont circulé comme celle du juriste Angelantonio Lanzellotti ${ }^{21}$. Cependant les grandes lignes en sont connues et semblent constituer un compromis entre les revendications portées par les libéraux depuis le decennio francese, l'exigence de maintenir la monarchie bourbonienne, en grande partie dictée par le contexte géopolitique, et l'impossibilité matérielle de rédiger en peu de temps une constitution adaptée au royaume. D'autant que la plupart des libéraux insistent sur la nécessité d'imiter les scénarios modernisateurs appliqués ailleurs; s'adressant aux Calabrais début août 1820, Guglielmo Pepe rappelle : «l'honneur de

realtà della Spagna, op. cit. Sur le parcours de Luis de Onís, cf. Didier Ozanam, Les diplomates espagnols au XVIII siècle, Madrid-Bordeaux, Casa de Velázquez-Maison des Pays ibériques, 1998, p. 366-7.

20. Voir notamment AHN, leg. 5674, n. 69, lettre de Luis de Onís à Evaristo Pérez de Castro, Naples, 27 septembre 1820 : «En una carta en cifra con fecha de 30 de Abril, participó el expresado sr. príncipe de Scilla, según se me ha referido, que yo había tenido otra reunión con mis amigos antes de mi partida de Madrid, en la cual había dado un brindis, a la propagación de las ideas liberales en Nápoles $[\ldots] »$.

21. Angelo Lanzellotti, Costituzioni politichi delle principali nazioni, Naples, 1820, 2 volumes. 
nos jours est de nous modeler à l'image de nos contemporains $»^{22}$. Chez des hommes qui ont largement soutenu l'éphémère République parthénopéenne de 1799, écrasée par la réaction sanfédiste, et ont globalement constitué des appuis du régime français de Murat, l'horizon républicain n'est, à de très rares exceptions près, pas à l'ordre du jour ${ }^{23}$. Par-delà des divergences d'opinion sur la manière de la mettre en place, en effet, la plupart des acteurs de la révolution s'accordent à en reconnaître la plus grande valeur de compromis que celle d'autres textes constitutionnels comme la Charte française de 1814, également connus des élites méridionales. Comme le signale, dans un organe de la presse libérale modérée, Matteo Galdi, ancien révolutionnaire de 1799, exilé en France, proche de Vincenzo Cuoco et appelé à devenir président du Parlement napolitain, le texte mérite d'être érigé en modèle car il est «sage, modéré, fils d'un savoir mûr et d'une expérience mûre» : il consiste "en un pacte social qui soustrait les peuples à la violence des gouvernements arbitraires $»^{24}$. Dès lors, le texte de Cadix offre une alternative cohérente au modèle politique alors en vigueur, la «monarchie administrative» autocratique, qui a été le principal héritage de l'ordre napoléonien en Italie et dont les structures ont été préservées par la monarchie légitimiste des Bourbons. Cette présentation contraste avec la lecture de certains libéraux espagnols au pouvoir qui jugeaient le texte trop révolutionnaire ${ }^{25}$, suivie par l'aile droite du courant libéral autour de Pietro Colletta et de Michele Carrascosa plutôt favorables au modèle britannique. Le texte de la constitution, qui met en place un système parlementaire monocaméral, ne remet cependant pas en cause le principe monarchique; il offre également une garantie d'indépendance et de souveraineté nationale sans réduire le rôle social de la religion, puisque la nation napolitaine est d'abord envisagée comme catholique. L'identification de la nation à un groupe confessionnel exclusif dirigé par un roi défini comme catholique est directement récupérée de la charte de Cadix $^{26}$. La principale vertu du texte choisi est donc de concilier les libéraux modérés et des patriotes plus radicaux en accordant aussi les partisans de la solution française, très nombreux à Naples parmi les anciens serviteurs du roi Murat. Dans la rhétorique libérale napolitaine, le texte de Cadix est donc réduit à ses dispositions institutionnelles générales qui reviennent à instaurer une monarchie parlementaire monocamérale, censitaire et catholique, reposant sur un espace public élargi.

22. Guglielmo Pepe, réponse aux Calabrais, citée dans Giornale Costituzionale del Regno delle Due Sicilie, n $^{\circ} 24$, vendredi 4 août 1820.

23. Guglielmo Pepe, Memorie, Paris, Baudry, 1847, volume 2, p. 58.

24. Dans L'Indipendente, 3 octobre 1820, p. viII.

25. Sur ce point, voir Jean-Philippe Luis, «Le premier constitutionalisme espagnol et la circulation des modèles politiques dans l'Europe du début du XIX e siècle», in Landry Charrier, Karine Rance, Friederike Spitzl-Dupic [dir], Circulations et réseaux transnationaux en Europe (XVIII $-X X^{e}$ siècles), Berne, Peter Lang, 2013, p. 89-102.

26. Cf. José Maria Portillo Valdés, La Nazione cattolica, op. cit., p. 91-113. 
Cette théorie de l'imitation en politique repose donc sur des rapports de fraternité entre les révolutions libérales méditerranéennes, idée héritée de la Révolution française et qui constitue un élément important de la culture politique libérale au XIX ${ }^{e}$ siècle ${ }^{27}$. La communication politique libérale appuie le choix de la constitution de Cadix et de l'imitation de la révolution d'Espagne en invoquant la fraternité libérale, fondée sur l'idée d'une communauté d'intérêts. Les premiers développements de la presse napolitaine, dès la mi-juillet 1820 , en témoignent, à travers la glorification systématique de l'expérience libérale espagnole, alors érigée en modèle. Un des grands organes de la presse libérale napolitaine, la Minerva Napoletana, évoque en août 1820 le "voyage politique» que les deux nations révolutionnées doivent mener ensemble ${ }^{28}$. Celui-ci procède en effet d'une cause commune, la régénération morale et politique, et profite de la sensibilité développée au problème espagnol et à la cause de l'indépendance par des vétérans partis combattre sur place pendant la guerre d'indépendance d'Espagne (1808-1814), comme Orazio De Attellis. Cette guerre constitue alors un topos de la mémorialistique libérale, à la fois par son caractère traumatique dû à l'ampleur et à la longueur d'un combat lointain et par le modèle qu'elle a fourni aux guerres de libération européennes en permettant l'autodétermination nationale, le refus de l'occupation étrangère et en constituant un terrain d'expression privilégié à la modernité politique naissante. D'autre part, les relations épistolaires entre les protagonistes des deux révolutions sont médiatisées dans la presse napolitaine. En août-septembre 1820, plusieurs journaux libéraux retranscrivent des lettres adressées à Guglielmo Pepe par les sociétés patriotiques d'Albuquerque, de Murcie et de Madrid, témoignant de l'engouement immédiat des révolutionnaires espagnols pour la cause napolitaine ${ }^{29}$. D'autres sont distribuées à Naples sous la forme de feuilles volantes, assimilant les sociétés patriotiques espagnoles à des sectes de carbonari, structure pourtant presque absente du paysage révolutionnaire espagnol ${ }^{30}$. Tous ces textes développent les solidarités entre les deux révolutions, scellées par l'adoption commune du texte gaditain dont on rappelle, à la suite des révolutionnaires espagnols, la portée universelle. À ce titre, la révolution de 1820 ne fait que continuer une histoire partagée depuis le Moyen Âge, expliquant la dette du royaume de Naples envers l'Espagne. Le jacobin Gioacchino Maria Olivier-Poli, patriote

27. On renverra sur ce point aux travaux du groupe ANR «Fraternités» : notamment Catherine Brice, Gilles Bertrand, Gilles Montègre [dir.], Fraternité : pour une histoire du concept, Grenoble, Cahiers du CRHIPA, 2012.

28. Minerva Napoletana, tome I, 1820, p. 32.

29. Plusieurs de ces lettres sont retranscrites dans les Annali del patriottismo, fascicule 1, 1820. La correspondance avec les révolutionnaires de Madrid est citée dans le Giornale costituzionale, $\mathrm{n}^{\circ} 72$, 29 septembre 1820 .

30. Cf. notamment BNN, SM, Fogli volanti, SQ IV, L. 28 n. 175, cité. La lettre, datée du 3 août 1820, fait de la constitution d'Espagne «il forte vincolo che stringerà la fraterna unione di due nazioni libere e indipendenti ». Sur les sociétés patriotiques, on renverra au texte classique d'Alberto Gil Novales, Las sociedades patrióticas (1820-1823) : las libertades de expresión y de reunión en el origen de los partidos politicos, Madrid, Tecnos, 1975. 
pendant la révolution de 1799, fait l'éloge de la «Nation espagnole, dont les habitudes et les coutumes ressemblent tellement aux nôtres " ${ }^{31}$, dans une réflexion à chaud sur les débuts de la révolution. La présence de deux dynasties espagnoles sur le trône napolitain jusqu'en 1734 et les liens familiaux que le roi bourbon entretient avec la royauté espagnole expliquent en effet un vaste héritage historique, culturel et politique qui légitime la réclamation de la constitution espagnole pour représenter les sujets du royaume de Naples. La construction d'un consensus autour du modèle espagnol apparaît néanmoins plus complexe, surtout dans les provinces, et s'appuie sur plusieurs catégories d'acteurs dont chacun donne à cette expérience un sens spécifique.

\section{Passeurs et Circuits De la politisation : Militaires, NOTABLES ET PRÊTRES}

Le déroulement des premiers jours de la révolution illustre les circuits empruntés par la politisation libérale avec l'objectif d'imposer le modèle espagnol dans les provinces. La présence des militaires, le rôle social des notables et des prêtres sont alors déterminants. Les grandes lignes de la diffusion des révolutions du premier $\mathrm{XIX}^{\mathrm{e}}$ siècle sont connues à la fois à l'échelle nationale et transnationale ${ }^{32}$. De ce point de vue, la majorité de la documentation concerne la province du Principat Ultérieur, autour d'Avellino, particulièrement impliquée dans la révolution et dont elle constitue un observatoire privilégié33. La chronique du juriste et lettré Biagio Gamboa, qui a été l'une des principales sources d'information de la police bourbonienne après la réaction malgré son ambition au départ mémorielle, décrit avec précision le rôle de ces acteurs dans la conversion de la province au modèle espagnol au point d'en faire le "théâtre de la régénération du royaume " ${ }^{34}$.

La contribution la plus souvent rappelée, parce que la plus déterminante pour la mise en œuvre matérielle de l'insurrection, est celle des militaires ${ }^{35}$. À

31. Gioacchino Maria Olivier-Poli, Cenno istorico sulla rigenerazione dell'Italia meridionale in luglio 1820, Naples, 1820, p. 19.

32. Ils sont l'objet de réévaluations récentes. Par exemple : Jean-Numa Ducange, Michel Biard [dir.], Passeurs de Révolution. Actes de la journée d'études organisée à Rouen le 14 janvier 2013, Paris, Société des études robespierristes, 2013; envisageant la spécificité des espaces ruraux : Julien Bouchet, Côme Simien [dir.], Les passeurs d'idées politiques nouvelles "au village», de la Révolution française aux années 1930, Clermont-Ferrand, Presses Universitaires Blaise-Pascal, sous presse.

33. Cf. les travaux, anciens mais érudits, de Vincenzo Cannaviello, Gli Irpini nella rivoluzione del 1820 e nella reazione, Avellino, Pergola, 1940, où l'on trouvera notamment la liste des hommes ayant pris part à la révolution. Sur Lorenzo De Conciliis, militaire du decennio francese qui est l'un des principaux acteurs de la révolution à Avellino, cf., id., Lorenzo De Concilj o Liberalismo Irpino, Naples, Fierro, 1913.

34. Biagio Gamboa, Storia della rivoluzione di Napoli entrante il Luglio del 1820, Naples, Trani, 1820, p. 9.

35. La mémoire collective locale a principalement retenu le rôle de Michele Morelli et de Giuseppe Silvati, pour leur rôle dans le déclenchement de l'insurrection. Voir essentiellement Mariano D’Ayala, I primi quattro martiri della libertà italiana nell'anno 1821, Naples, s.n., 1861, p. 3-6 (pour Morelli), p. 17- 22 (pour Silvati). 
ceux-ci, l'expérience espagnole a fourni des modèles d'héroïsme, comme l'attestent les parallèles fréquemment établis entre l'Espagnol Rafael del Riego et le Napolitain Guglielmo Pepe, tous deux généraux, et surtout entre Antonio Quiroga et Lorenzo De Conciliis, les officiers à l'origine du déclenchement des deux révolutions ${ }^{36}$. Gamboa rappelle comment Luigi De Francesco, ancien jacobin et juge à Avellino, aurait invité le major De Conciliis à suivre l'exemple du général espagnol, le royaume des Deux-Siciles ayant besoin "d'un militaire déterminé " ${ }^{37}$. Dès lors, De Conciliis a fortement contribué à recruter dans les villages de la région d'Avellino les militaires, professionnels ou non, qui constituent le premier bataillon engagé dans la révolution, engageant la révolte à Avellino un jour avant la généralisation de l'émeute. Ces hommes ont pour la plupart été recrutés dans le large réseau des sociétés secrètes auxquelles De Conciliis n'appartient pas, mais dont, par sa fonction de notable local, il connaît les membres les plus influents. Cela lui permet d'imposer que ne soient soldats que «les seuls amis de la patrie ${ }^{38}$. Chef d'état-major de la province d'Avellino, De Conciliis avait envoyé plusieurs militaires se charger du recrutement dans leurs villages d'origine, comme Nunziante Preziosi ou Modestino Bianchi à Mercogliano, en périphérie proche d'Avellino. À Avella, à la limite occidentale de la province, il avait envoyé deux autres militaires, Luciani et Pepare. Ce sont les mêmes émissaires qui imposent le drapeau révolutionnaire et la constitution d'Espagne dans d'autres provinces, comme le capitaine Gaetano Lente dans plusieurs villages de Terra di Lavoro, au nord d'Avellino ${ }^{39}$. Ces émissaires permettent de former, le $1^{\text {er }}$ juillet 1820 , un bataillon de patriotes locaux dont la composition est connue, 25 officiers et 94 soldats, très majoritairement militaires de métier ${ }^{40}$. Ce sont eux qui rejoignent Michele Morelli et Giuseppe Silvati le 2 juillet dans la garnison de Nola et constituent les premiers effectifs de l'Armée constitutionnelle formée par Guglielmo Pepe le 6. On perçoit, à travers la mobilisation des hommes, comment les officiers napolitains utilisent les ressorts du pronunciamiento espagnol, outil majeur des révolutions libérales des années 1810-1820 qui consiste à armer le peuple et à proclamer ses revendications ${ }^{41}$. À l'exemple des corps militaires espagnols donc, l'armée constitutionnelle est pensée à la fois comme l'initiatrice et le rempart du régime libéral chez des acteurs qui gardent en tête l'écrasement de la révolution de 1799 par les sanfédistes. Le défilé organisé par Pepe dans les rues de

36. Parmi la littérature immédiate : Biagio Gamboa, Storia della rivoluzione di Napoli, op. cit., passim; Orazio De Attellis, L'Ottimestre costituzionale delle Due Sicilie, op. cit., passim.

37. Biagio Gamboa, Storia della rivoluzione di Napoli, op. cit., p. 90.

38. Ibidem, p. 9.

39. Rapporto del capitano Gaetano Lente a De Conciliis, Vitolano, 7 juillet 1820, cité par Biagio Gamboa, Storia..., op. cit., p. 35, appendice XXV.

40. On dispose d'états nominatifs précis des officiers et des soldats recrutés par De Conciliis (BSNSP, mss. 15529).

41. Sur la politisation des militaires espagnols et la pratique du pronunciamiento : José Cepeda Gómez, El ejército español en la política española 1787-1843. Conspiraciones y pronunciamientos en los comienzos de la España liberal, Madrid, Fundación Universitaria Española, 1990, p. 148-224. 
Naples le 9 juillet témoigne de l'importance symbolique de ce corps d'armée. Dès les premiers jours de la révolution, Pepe adresse plusieurs proclamations au peuple napolitain, appelle la nation à s'armer et entend distinguer les soldats "les plus méritants" par deux commissions composées des principaux officiers du royaume ${ }^{42}$. Comme en Espagne, ce processus de distinction permet de faire émerger de nouvelles figures hérö̈ques qui complètent le panthéon national principalement composé des Irpiniens et des Samnites, qui se revendiquent héritiers des armées romaines de l'Antiquité et dont les qualités militaires et patriotiques sont très largement vantées par des feuilles volantes distribués dans la capitale. Un thuriféraire de la révolution comme Biagio Gamboa fait le parallèle entre le défilé de l'armée constitutionnelle et les triomphes romains ${ }^{43}$. Ce processus d'héroïsation et de médiatisation de l'armée rejoint des constructions intellectuelles autour de la «nation en armes», comme celle développée par le publiciste Domenico Duccilli dans le Liceo Costituzionale, l'un des périodiques majeurs de l'aile libérale napolitaine dont il est le contributeur principal ${ }^{44}$. Le thème relève d'une rhétorique révolutionnaire plus large qui emprunte à l'expérience française et vise à constituer des milices de citoyens-patriotes. Néanmoins, l'assise sociale insuffisante de la révolution explique les difficultés rencontrées par les chefs libéraux pour lever des effectifs suffisants. On perçoit donc, à travers l'incapacité à mobiliser des troupes coordonnées pour défendre et imposer la constitution dans le royaume, la difficulté à transposer à Naples le modèle révolutionnaire espagnol fondé sur l'insurrectionnalisme.

À un moment où les militaires ne constituent pas une catégorie sociale clairement individualisée - le langage administratif est, de ce point de vue, flottant, rendant souvent le rôle militaire secondaire au statut de propriétaire ou à l'appartenance à la noblesse ${ }^{45}$-, c'est d'abord par leur statut de notables et par leur influence sociale à l'échelle locale que les principaux chefs libéraux parviennent à imposer la constitution d'Espagne et à lever des troupes pour la défendre. Ainsi, le rôle décisif de Lorenzo De Conciliis dans la conversion du Principat ultérieur à la constitution d'Espagne s'explique en très grande partie par le poids de sa famille dans le paysage économique et social d'Avellino où elle est implantée depuis le XVIII siècle, pratiquant le négoce, possédant l'un des plus grands palazzi de la ville. Ses quatre frères, Matteo, Felice, Filippo et Tommaso, notables insérés dans les réseaux des sociétés secrètes, exercent une influence locale très forte ${ }^{46}$. De la même manière, Nicola Imbimbo, l'un

42. Notamment Guglielmo Pepe, lettre au duc de Calabre, 16 juillet 1820, citée dans Ruggiero Moscati, Guglielmo Pepe, tome 1, Roma, Vittoriano, 1938, p. 69-70.

43. B. Gamboa, Storia ..., op. cit., p. 109.

44. Domenico Duccilli, "Della milizia nazionale», Liceo Costituzionale, III, 1, 15 sept. 1820, p. $182-194$.

45. Cf. Anna Maria Rao, Esercito e società nell'età rivoluzionaria e napoleonica, Naples, Morano, 1990.

46. $C f$. les observations régulières envoyées par les consuls des pays d'accueil au ministère napolitain des Affaires étrangères: Archivio di Stato di Napoli (noté par la suite ASN), Interno, 40, 
des officiers qui ont proclamé la constitution à Avellino début juillet 1820, est recensé comme propriétaire et appartient, avec ses deux frères Giuseppe et Tommaso également impliqués dans la révolution, aux notables de la province $^{47}$. Eux aussi sont issus d'une importante famille marchande installée à Avellino depuis le $\mathrm{XVI}^{e}$ siècle. Biagio Gamboa rappelle les efforts mis en place par De Conciliis et Imbimbo auprès des propriétaires de la région lésés par la politique fiscale des Bourbons. Par l'intermédiaire de ces hommes largement insérés dans les réseaux des sociétés secrètes locales, ils associent les revendications fiscales, dont l'historiographie marxiste a montré le rôle central dans le déclenchement de la révolution, à la réclamation d'une constitution politique ${ }^{48}$. Ces riches propriétaires jouent un rôle déterminant dans la levée des troupes révolutionnaires à l'échelle locale. Aux rapports d'influence économique s'ajoutent des rapports politiques : c'est ce qui explique l'implication de plusieurs fonctionnaires, comme le procureur général d'Avellino Giuseppe Marini ou le conseiller d'intendance Pietro De Luca, qui constituent des relais importants du modèle espagnol auprès des administrations dont ils contribuent de façon décisive à la politisation libérale. À l'échelle locale, plusieurs maires, comme Modestino Bianchi à Mercogliano, proclament la constitution dans leur village, érigent le drapeau révolutionnaire et fournissent des moyens et des vivres à l'armée constitutionnelle ${ }^{49}$.

À côté des élites politiques et économiques locales, les prêtres ont également constitué un canal important d'imposition de la constitution d'Espagne dans les provinces. Le cas le plus emblématique est celui de Luigi Minichini, curé de Nola où il est, sous le nom d'emprunt d'Antonio Montano, l'un des initiateurs du soulèvement du 2 juillet 1820, profitant de sa fonction de grand maitre carbonaro de la vente locale ${ }^{50}$. Coordinateur du mouvement militaire aux côtés de Michele Morelli et Giuseppe Silvati, correspondant régulier des chefs de la révolution dont Pepe et Carrascosa, il est surtout l'auteur de l'une des principales chroniques de l'insurrection de l'été $1820^{51}$. Son rôle n'est pas isolé : le personnel clérical, au moins séculier ${ }^{52}$, a joué un rôle mobilisateur important. Les sources de la répression en 18211822 en montrent la très forte présence dans les sociétés secrètes libérales au point que, dans certains villages du sud-est de la province comme à Andretta

Registro degli espatriati e esuli del Regno delle Due Sicilie, «De Concilj (Matteo)», «De Concilj (Felice)», «De Concilj (Filippo)», «De Concilj (Tommaso)».

47. BSNSP, mss. 115529.

48. B. Gamboa, Storia..., op. cit., p. 15. Parmi les travaux qui ont rappelé les moteurs économiques de la révolution de 1820-21, on retient surtout celui d'Aurelio Lepre, La rivoluzione napoletana del 1820-1821, Roma, Editori Riuniti, 1967.

49. B. Gamboa, Storia..., op. cit., p. 18, et surtout ASN, Interno, 40, cit., «Bianchi (Modestino)».

50. ASN, Interno, 40, cit., «Minichini (Luigi)».

51. Luigi Minichini, Luglio 1820. Cronaca di una rivoluzione, Roma, Bulzoni, 1979.

52. Alors que le clergé régulier est beaucoup moins impliqué en politique, lorsqu'il n'est pas légitimiste. Cf. Ugo Dovere, «I francescani napoletani di fronte al Risorgimento", Studi francescani, $n^{\circ} 109 / 3-4,2012$, p. 415-439. 
ou à Teora, la quasi-totalité des prêtres appartient à la Charbonnerie et a contribué à la proclamation de la constitution ${ }^{53}$. Cette tendance se retrouve, quoique dans des proportions moindres, sur la totalité de l'itinéraire routier et postal Avellino-Potenza, aux confins méridionaux de la province, voisine de celle de Salerne où a été introduite la nouvelle de la révolution d'Espagne. Les plus haut gradés, considérés les plus dangereux par les sources de police, ont entre 45 et 50 ans et ont donc connu la révolution de 1799 et l'occupation française. Près d'Avellino, Domenico Ferrara diffuse la constitution autour de la paroisse de San Mango dont il a la charge. À Sant'Angelo a Scala, Nicola Simeone prédit dès les premiers mois de 1820 l'avènement de la constitution d'Espagne. À Montefusco au nord d'Avellino, Felice Isauro Urciuoli explique l'intérêt de la constitution d'Espagne dans un tract destiné au peuple, envoyé le 9 juillet. Beaucoup de ces prêtres se sont rendus à Naples pour assister au défilé de l'armée constitutionnelle le 6 juillet, comme Benedetto Di Rienzo, chanoine à Pietradefusi, également souscripteur de bons de financement du corps armé libéral après avoir imposé la constitution dans son village avec trois prêtres, Luca Nardone, Antonio Petitto et Abele De Cristofaro. D'autres, comme le chanoine Andrea Nazzaro de Manicalciati, souscrivent plusieurs bons à Avellino, à Nola et à Naples. La participation des prêtres au fonctionnement de l'armée constitutionnelle révèle les interférences entre le clergé et le monde militaire, tous deux acteurs de la révolution. Par-delà les logiques locales et le poids des réseaux d'opposition, l'importante participation des prêtres à la révolution, courante dans le monde libéral de l'époque, s'explique en grande partie par leur adhésion à un programme dont l'ambition est de former une nazione cattolica, construisant une voie originale du libéralisme rendu compatible avec le christianisme ${ }^{54}$. Les sermons de l'archevêque de Catanzaro (Calabre Ultérieure II), publiés à Messine au début de l'année 1821 et largement diffusés en Calabre, vont dans ce sens, se proposant de concilier le libéralisme gaditain avec la théorie chrétienne du pouvoir telle qu'elle est, notamment, formulée par saint Paul ${ }^{55}$. L'exemple n'est pas isolé et pose la question de l'adaptation plus large du texte de Cadix aux conditions propres au royaume des Deux-Siciles, objet d'un important débat qui mobilise à la fois la société civile napolitaine et plusieurs juristes et observateurs internationaux.

53. ASN, Polizia generale, 4574, Stato nominativo dei preti della provincia di Principato Ultra, 1822.

54. La participation des prêtres aux révolutions libérales est peu connue. On sait seulement qu'en Espagne métropolitaine et coloniale, les prêtres ont été nombreux à diffuser les thèses libérales par des sermons, à réfléchir au "libéralisme chrétien" ou à se mobiliser pour l'autonomie politique. Pour l'Espagne, voir Luis Barbastro Gil, «El "catolicismo liberal” de Villanueva, Bernabeu y Cortes. Una contribución decisiva al primer liberalismo (1808-1823)", Spagna contemporanea, 26, 2004/1, p. $1-24$.

55. Michele Basilio Clary, Il liberalismo cristiano. Omelie sacro-politiche recitate nella sua catedrale nell'avvento dell'anno 1821, Messine, Pappalardo, 1822. 


\section{LES FREINS À L'IMITATION}

Une fois le texte de Cadix adopté par Ferdinand Ir et imposé aux provinces napolitaines, un vaste débat s'ouvre dans l'espace public napolitain à propos de la "naturalisation" possible d'un texte de loi étranger à la législation et aux conditions politiques du royaume des Deux-Siciles ${ }^{56}$. Début août 1820, un journal libéral modéré comme la Minerva en fait l'une de ses priorités au point d'ouvrir son premier numéro sur la question de l'adaptation du texte : «La constitution rédigée pour l'Espagne, alors qu'elle était en son sein presque piétinée par l'envahisseur, alors que son roi endurait la prison, la constitution de Cadix est peut-être trop marquée par le moment et par les circonstances dans lesquels elle a été produite ${ }^{57}$. Un tel point de vue interroge le caractère légitime d'un texte constitutionnel importé et adopté tel quel. Pour les auteurs de la Minerva, il s'agit donc d'un texte conjoncturel qui prend son sens dans le contexte d'occupation militaire et d'aliénation de l'autorité monarchique dans lequel il a été écrit, qui n'est pas celui du royaume de Naples en 1820, pays souverain dirigé par sa propre dynastie. L’organe libéral exprime donc sa méfiance au point de considérer comme "servile» l'observation stricte du texte espagnol ${ }^{58}$. L'idée d'adapter la constitution espagnole est alors largement admise dans l'espace public napolitain et fait l'objet d'une partie importante des débats parlementaires ${ }^{59}$. Au moment de la publication de la constitution en effet, on observe le développement considérable des textes de glose de la part de juristes, de prêtres libéraux, voire de simples citoyens éclairés. On recense, sur la question, 26 titres écrits et publiés à Naples par des Napolitains. La plupart des améliorations portent sur la question religieuse, centrale dans le texte de Cadix, dont les auteurs regrettent qu'il définisse le royaume méridional comme une nazione cattolica, réalité qui pose le problème d'être inadaptée à la situation d'un pays qui a lourdement subi la contre-révolution au lendemain de l'expérience républicaine de 1799 et où le clergé, dans certaines provinces comme les deux Principats ou les trois Calabres, est très largement acquis à la cause libérale ${ }^{60}$. D'autres, beaucoup plus rares, s'interrogent sur la validité des institutions : en s'appuyant sur la constitution de Sicile de 1812, largement inspirée des

56. L'expression est empruntée à Leonidas Pépé, Lettres sur la dernière révolution de Naples, sur ses causes, ses cheff, ses incidences et sa catastrophe, Paris, 1821, I, p. 10.

57. Minerva napoletana, I, 1, août 1820, p. 4.

58. Ibidem, p. 16.

59. On trouvera un aperçu de ces derniers dans Alfonso Scirocco, «Parlamento e opinione pubblica a Napoli nel 1820-21 : l'“adattamento" della Costituzione», Clio, IV, 1990/4, p. 569-578.

60. Parmi les textes qui développent tout ou partie de cette perspective : Brevi riflessioni su miglioramenti essenziali che sarebbero necessari a farsi alla Costituzione Spagnola per addattarla allo sviluppo della Nazione del Regno delle Due Sicilie, Naples, Luigi Nobile, 1820; Riflessioni analitiche sullo stato attuale delle Due Sicilie. E mezzi che si propongono per una generale riforma, che possono valere di modificazione alla costituzione spagnuola, Naples, s.n., 1820; Carlo Mele, La Costituzione spagnuola esaminata secondo i principi della ragione, e modificata secondo le circostanze del regno delle Due Sicilie, Naples, De Bonis, 1820. 
institutions pourvues par l'occupant britannique par lequel elle a été dictée, Filippo Pagano plaide pour le modèle bicaméral ${ }^{61}$. Le débat trouve un écho à l'échelle internationale : des observateurs étrangers comme le juriste français Jean-Denis Lanjuinais ou le diplomate Dufour de Pradt produisent des gloses de la constitution, s'interrogent sur son caractère adapté ou non au cas napolitain; leurs écrits sont traduits en italien et republiés à Naples ${ }^{62}$. Ces textes connaissent un écho important dans les sociabilités politiques éclairées de la capitale méridionale.

Un des problèmes posés par l'adaptation du texte consiste à savoir s'il faut retraduire la constitution pour l'adapter aux réalités napolitaines, le Parlement ayant prévu une simple commission chargée, dès juillet 1820 , de vérifier la traduction de Masdeu. À cet égard, plusieurs articles émanent de citoyens dans un modèle politique qui laisse une large place à la participation. L'Imparziale et les Annali del patriottismo livrent tous les deux, en novembre 1820, le point de vue d'un médecin libéral, Vitangelo Morea, également disciple du poète Vincenzo Monti qui l'a formé à la littérature et aux langues étrangères, qui critique une traduction trop hâtive et interroge le caractère véritablement exportable d'institutions pensées au départ pour un pays étranger ${ }^{63}$. Le point principal de l'argumentation alerte les lecteurs sur le fait que la traduction proposée par le gouvernement s'est contentée de donner aux termes espagnols une forme italienne, ce qui pose des questions de cohérence à un moment où la pratique de la traduction littéraire se formalise et prend son sens moderne sous l'impulsion d'auteurs comme le chanoine Masdeu, attentifs au respect scrupuleux du style littéraire du texte original. Plusieurs notions, pour la plupart relatives aux communautés politiques (Corti; giunta) et au gouvernement local (aggiuntamento; alcalde; vecino; partito), sont jugées inadaptées aux réalités politiques et sociales d'un royaume profondément transformé par dix ans d'administration par l'occupant français, très différentes, donc, d'une monarchie centralisée qui peine à élaborer, après 1808, une convention politique alternative à la monarchie d'Ancien Régime. L'auteur relève également plusieurs absurdités : le terme junta, qui désigne une assemblée, aurait été traduit à tort par l'italien giunta, terme technique qui exprime un rajout en couture ou en cuisine. De la même manière, le vocable aggiuntamento, qui désigne le gouvernement municipal, serait dépourvu de sens politique et renverrait au terme aggiuntatore qui

61. Filippo Pagano, Osservazioni Critiche sulla Costituzione della Monarchia Spagnuola, Naples, s.d., 1820. Rappelons que la Sicile, a subi l'occupation militaire britannique au cours du decennio français alors qu'elle a servi de refuge à la dynastie bourbonienne. L'ordre impérial, incarné par le gouverneur général lord William Bentinck (1774-1839), a été marqué par l'imposition d'un texte constitutionnel reproduisant en grande partie les institutions britanniques.

62. Jean-Denis Lanjuinais, Considerazioni politiche su i cambiamenti da farsi alla Costituzione di Spagna per assodarla, specialmente nel Regno delle Due Sicilie. Opera del Sig. Lanjuinais recata in italiano dal sig. Filippo Marzullo, Napoli 1821; Dominique Dufour de Pradt, Esame della Costituzione spagnuola del sig.re De Pradt... Tradotta dal sig. R.B., Napoli, 1820.

63. Voir notamment Vitangelo Morea, «Osservazioni sulla traduzione offiziale della costituzione spagnuola, fatta in Napoli per ordine del Governo ", L'Amico della costituzione, III, 1820, p. 37-52. 
signifie escroc. Selon V. Morea, ces deux termes renverraient, de manière inopportune, au verbe giuntare (frauder). Il propose alors de substituer à ces transpositions hâtives des concepts dotés d'une réelle signification historique et culturelle pour des Italiens, comme le terme municipalità qui renvoie aux Communes médiévales ou ceux de congresso ou comizi qui font référence à la République romaine. En ce sens, les solutions envisagées par Morea glorifient les expériences politiques autonomes de l'histoire italienne dans le prolongement logique desquelles il situe la révolution constitutionnelle de Naples, négligeant que le royaume de Naples n'a pas connu de tradition communale comparable à celle de l'Italie du Nord ou de la Toscane. D'autant que la révolution de Naples n'introduit pas de structures de gouvernement local comparables à celles mises en place en Espagne. Le thème n'est pas nouveau à un moment où la production historique libérale - et pas seulement napolitaine - appuie ses observations sur la recherche de précédents historiques et sur la référence à des ancêtres mythifiés ${ }^{64}$. Du point de vue des traducteurs donc, le texte constitutionnel gaditain apparaît inadapté car il conduit à plaquer des concepts politiques espagnols sur des réalités napolitaines très différentes.

\section{D’AUTRES MODÈLES}

Des publicistes napolitains confrontent donc le texte de Cadix à plusieurs autres constitutions contemporaines, à la recherche d'une solution mieux adaptée. Alors même que le texte est adopté par le gouvernement provisoire le 9 juillet 1820, on voit circuler d'autres constitutions, également traduites en italien et publiées à Naples : les constitutions imprimées sont alors un objet de librairie courant. Une librairie comme la maison R. Marotta e Vanspandoch, située piazza San Domenico Maggiore dans le centre de Naples, a en vente 6291 textes constitutionnels en mars 1821, dont près de la moitié ont été produits par la France révolutionnaire et impériale. Seulement 7\% du fonds de la librairie est composé de constitutions napolitaines du nonimestre. Significativement, la librairie ne vend aucune traduction du texte de Cadix, alors même qu'elle propose 400 exemplaires de la constitution accordée par Joseph en 1808 et 500 catéchismes politiques de $1812^{65}$. Les constitutions, et plus largement l'actualité politique étrangère sont largement commentées par la presse qui s'attarde sur les questions institutionnelles. Le juriste Guglielmo Palladino (1774-1840) fait l'éloge du modèle anglais. Un autre juriste, Angelo Lanzellotti (1782-1833), publie, pour « rendre son plein ser-

64. Cette pratique propre à l'écriture historique et mémorielle du XIX e siècle a été montrée, pour la France, par Sylvain Venayre, Les origines de la France. Quand les historiens racontaient la nation, Paris, Seuil, 2013. Sur l'Italie du XIX ${ }^{\mathrm{e}}$ siècle, on trouvera quelques éléments de réflexion dans Alberto Mario Banti, Roberto Bizzocchi [dir.], Immagini della nazione nell'Italia del Risorgimento, Roma, Carocci, 2002, notamment A.M. Banti, "Le invasioni barbariche e le origini delle nazioni», p. 21-44.

65. Archivio di Stato, Livorno, Auditore del Governo, a. 1821, n. 603. 
vice à la patrie ${ }^{66}$, un recueil de constitutions napolitaines et européennes en deux volumes : l'objectif est de choisir le texte le plus adapté au cas napolitain. Le débat est relayé à l'étranger, outre les commentaires de juristes sur la constitution de Cadix, par l'influence intellectuelle de penseurs étrangers comme Benjamin Constant et Jeremy Bentham. La grande majorité des propositions se fondent sur le critère de l'utilité pour le régime napolitain. L'ensemble des auteurs développent, outre les conditions historiques, politiques et sociales du royaume, les caractères géographiques et culturels qui le différencient de l'Espagne et justifient la réclamation d'un texte adapté. Il faut y voir l'influence décisive de la pensée politique napolitaine du XVIII siècle et plus précisément des réflexions de Vincenzo Cuoco sur la révolution de 1799, alors récemment republiées sous la direction du général Pietro Colletta. La thèse de Cuoco, selon laquelle l'échec de la révolution s'explique par ses structures importées et largement inadaptées aux conditions locales du royaume des Deux-Siciles - négligeant, essentiellement, la très faible éducation politique du peuple méridional - rejoint en effet la théorie développée depuis Giambattista Vico selon laquelle la modernisation économique ou politique ne peut être harmonieuse que si elle procède des "vocations territoriales" : cette conception déterministe, très largement partagée par les libéraux napolitains du XIX ${ }^{\mathrm{e}}$ siècle, s'exprime à l'échelle locale ${ }^{67}$. À cet égard, et au même titre que la Révolution française de 1789, la révolution espagnole du trienio ne saurait être importée sans adaptation au royaume des DeuxSiciles.

De ces mêmes réflexions découle la nécessité d'éduquer le peuple napolitain à la révolution : le souvenir traumatique de la révolution de 1799, "manquée», selon les termes de V. Cuoco, en l'absence d'une assise populaire suffisante. L'explosion des publications de feuilles volantes, d'opuscules développant des concepts politiques simplifiés a profité, comme l'a noté Werner Daum, de la libéralisation de la presse et de l'imprimerie à l'occasion du nonimestre constitutionnel ${ }^{68}$. Les schémas de communication employés sont empruntés au modèle espagnol. Dès les premiers jours de 1820 est publiée à Naples l'édition italienne du catéchisme officiel de la monarchie espagnole ${ }^{69}$. Alors que l'usage des catéchismes politiques est admis - il était déjà pratiqué lors de la révolution de 1799 qui a vu circuler des catéchismes français -, leur caractère importé suscite des réticences comparables à celles provoquées par la constitution de Cadix. En août 1820, le premier numéro de la Minerva Napoletana, organe de presse d'élite lu par des libéraux fortement imprégnés

66. Angelo Lanzellotti, Costituzioni politichi delle principali nazioni, op. cit, p. Iv.

67. Voir Marta Petrusewicz, "La modernizzazione che venne dal Sud", in Marta Petrusewicz, Jane Schneider, Peter Schneider [dir.], I Sud: conoscere, capire, cambiare, Bologne, il Mulino, 2009, p. $105-130$.

68. Werner Daum, Oszillationen des Gemeingeistes. Öffentlichkeit, Buchhandel und Kommunikation in der Revolution des Königreichs beider Sizilien, 1820/21, Cologne, SH-Verlag, 2005.

69. Catechismo costituzionale della monarchia spagnuola. Destinato ad illuminare il popolo, ad istruire la gioventù ed all'uso delle scuole primarie. Prima traduzione italiana, Naples, s.n., 1820. 
des réflexions de V. Cuoco, exprime ses réserves à propos d'un livre dont « [l] a lecture donne à tout Napolitain la nausée et suscite chez lui l'indignation ", là encore, en raison de décalages entre les conceptions véhiculées par l'ouvrage et les réalités méridionales ${ }^{70}$. C'est ce qui explique que le gouvernement provisoire napolitain en vienne à charger une commission de la rédaction d'un catéchisme officiel qui, significativement, se démarque dès les premières lignes du texte espagnol par la référence à la liberté religieuse ${ }^{71}$. Trois autres textes produits par des citoyens développent des arguments comparables. Des auteurs en viennent à expliquer au peuple, par l'intermédiaire de feuilles volantes anonymes à fort tirage, la validité exclusive des textes politiques pour le pays qui les a produits. Un dialogue fictif en circulation à Naples à la fin de l'année 1820, anonyme et sans indication d'éditeur, impliquant un Napolitain et un Espagnol, fait reconnaître au second que le royaume des Deux-Siciles a besoin de construire sa propre révolution, indépendamment des modèles étrangers ${ }^{72}$. L'usage de canaux spécifiquement conçus pour un peuple inégalement alphabétisé et inégalement italophone le confirme : les chiacchieriate, dialogues politiques en dialecte, se chargent d'expliquer les concepts du libéralisme politique en invoquant des réalités quotidiennes connues des Napolitains. Mais cet espace public constitué par les journaux, les revues et les tracts reste largement limité à la capitale; leur diffusion ne touche que très peu les provinces où, à quelques exceptions près - les deux Principats, les trois Calabres et la Capitanate essentiellement - les révolutionnaires demeurent minoritaires. La faible implantation sociale du libéralisme napolitain est en grande partie ce qui explique l'échec du mouvement de 1820-1821.

Malgré l'implication décisive de plusieurs catégories de passeurs qui ont réussi à imposer le modèle espagnol jusque dans certaines provinces napolitaines, les législateurs napolitains se sont donc rapidement heurtés au caractère inadapté d'institutions importées, dont la transposition dans le royaume des Deux-Siciles apparaît complexe. D’autant que la postérité de ce transfert est limitée : une fois la révolution réprimée par l'armée autrichienne après mars 1821, c'est davantage par son caractère antibourbonien que par son caractère importé que l'épisode révolutionnaire de 1820-21 apparaît illégitime. Les autorités y voient alors plutôt un moment de l'opposition des libéraux à la monarchie au même titre que la révolution de 1799 et le régime d'occupation française dont la révolution aurait été l'héritière directe. De la

70. Minerva Napoletana, I, 1, op. cit., p. 23.

71. Catechismo costituzionale. Per uso del Regno unito delle Sicilie, Naples, Sangiacomo, 1820.

72. Discorso politico tra lo Spagnuolo, e Napoletano di N.P.D.A.F., Naples, s.n., 1820: «Le Costituzioni de'Stati debbonsi fondare a seconda de'costumi de'paesi pei quali le stesse servono". 
même manière, la littérature libérale postérieure à l'effondrement du régime révolutionnaire espagnol en 1823 perd de vue le modèle gaditain. En faisant de l'importation de la constitution d'Espagne un aspect secondaire d'une insurrection dominée par les sociétés secrètes et par le retour des héritages du Premier Empire, elle montre à quel point cette hispanophilie a été conjoncturelle et opportuniste ${ }^{73}$.

\author{
Pierre-Marie Delpu est agrégé d'histoire \\ et doctorant contractuel \\ à l'université Paris 1 - Panthéon-Sorbonne.
}

73. L'opportunisme des hommes de 1820-1821 est l'un des grands thèmes de la littérature napolitaine quarante-huitarde. Cf. par exemple Giuseppe Massari, I casi di Napoli dal 29 gennaio 1848 in poi. Lettere politiche, Turin, Ferrero e Franco, 1849, passim. 